\title{
Substantiation of the Necessity for Design of Geohod Control System
}

\author{
Vladimir Aksenov 1,3*, Ivan Chicherin ${ }^{2}$, Irina Kostinez ${ }^{4}$, Anton Kazantsev ${ }^{3}$, and Andrey \\ Efremenkov ${ }^{3}$ \\ ${ }^{1}$ The Federal Research Center of Coal and Coal-Chemistry of Siberian Branch of the Russian \\ Academy of Sciences, 650610, 10 Leningradski av., Kemerovo, Russia. \\ ${ }^{2}$ T.F. Gorbachev Kuzbass State Technical University, 650000, 28 Vesennyaya St., Kemerovo, Russia \\ ${ }^{3}$ Yurga Technological Institute branch of Tomsk Polytechnic University, 652052, 26 Leningradskay \\ str., Yurga, Russia \\ ${ }^{4}$ T.F. Gorbachev Kuzbass State Technical University, Belovo Branch, 652644 Ilyicha st. 32, Inskoy, \\ Kemerovo oblast, Russia
}

\begin{abstract}
The article proves the necessity of creating a control system for the geohod. The scope of application of the geohod is described. The general scheme of the geohod with the main structural elements - cutter drum, head and tail sections, external mover - is described. Enlarged tasks that need to be addressed when creating a control system for the geohod are identified. The tasks of motion control of the geohod in the geomedium, improving the efficiency of the geohod, positioning the geohod in space, control of the support systems, diagnostics of the condition of the geohod, interaction of the control system with the operator are described. The directions of further research are identified: to formulate the requirements for the geohod control system; to analyze tasks solved by the geohod control system; develop the structure, mathematical models and algorithms for the operation of subsystems of the geohod control system; to develop software to simulate the interaction of subsystems of the geohod control system with each other, with the geomedium and the operator.
\end{abstract}

\section{Introduction}

Geohod technology is a promising method of mining [1,2,3]. A technical tool that implements this method is the geohod. A geohod is a machine that uses the geomedium to move in an underground space. Geohod fields of application are very wide $[4,5,6,7]$ :

- mine working penetration of various locations in space;

- building of underground facilities for various purposes;

- laying of the city's sewer line;

\footnotetext{
*Corresponding author: 55vva42@mail.ru
} 
- construction of the main metro tunnels;

- construction of underground warehouses, storage facilities, underpasses, garages;

- conducting emergency rescue operations in the rubble;

- construction of field fortifications.

The construction of the geohod is made on a modular basis (figure 1). The basic components of the geohod are the executive body, which destroys coalface (cutter drums), head and tail sections, the external mover $[8,9,10,11]$.

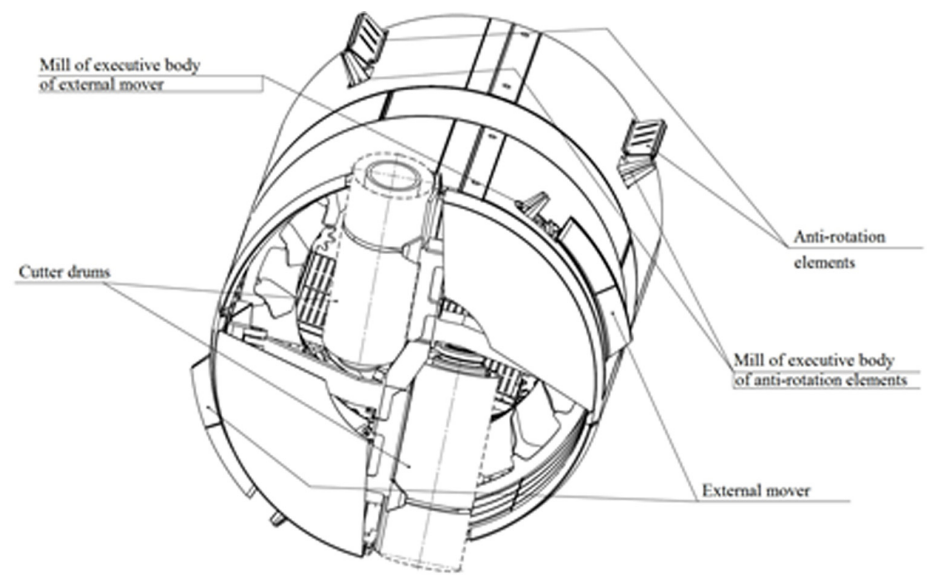

Fig. 1. General view of the geohod.

For effective work of the geohod, a clear interaction of all systems of the geohod is required, in particular, taking into account changing conditions in the external geomedium. To ensure such interaction a control system is required that will allow to control the particular systems and the geohod in general.

Tasks solved by the geohod control system. To ensure effective work of the geohod, it is necessary to create a control system that implements many tasks. The list of these tasks depends on technical characteristics of a particular geohod model. However, all of these tasks can be divided into the following classes:

1) motion control of the geohod in the geomedium;

2) improving the efficiency of the geohod;

3) positioning of the geohod in space;

4) control of auxiliary systems;

5) diagnostics of the state of the geohod;

6 ) interaction of the geohod control system with the operator.

The task of the geohod motion control, in the first approximation, is the task of controlling executive mechanisms, the basis of which are hydraulic cylinders and hydraulic motors. The hydraulic cylinders and hydraulic motors drive the executive bodies, as a result of which the geohod moves in the geomedium $[12,13]$. This task can be carried out both in manual and automatic modes. In manual mode, an operator with the help of controls turns on the executive mechanisms and changes the parameters of their work for maneuvering and changing the speed of geohod movement. In automatic mode, the operator specifies the direction and the speed of movement, and the control system calculates the operation parameters of the executive mechanisms and applies the appropriate control actions.

The task of improving the geohod efficiency is divided into two subtasks:

- improving the geohod efficiency based on optimization criteria;

- improving the geohod efficiency based on the use of adaptive algorithms that take into account the heterogeneity of the geomedium and dynamic parameters of the geohod. 
To solve the optimization problem, it is necessary to develop mathematical models and intelligent algorithms [14] for the operation of the geohod control system, taking into account the interrelation of all its elements. These models and algorithms will allow to apply to the executive mechanisms such control actions, in which the work of the geohod will satisfy the specified optimization criterion. The optimization criteria can be the geohod energy consumption per unit path, the accuracy of the trajectory.

Mathematical models for the adaptive control should take into account the heterogeneity of the parameters of geological medium (Protodyakonov scale of hardness, etc.) [12] and dynamic parameters of the geohod, which will depend on the speed, acceleration, direction (right, up, down, left, right) of the geohod.

Currently, the task of orientation of ground objects is effectively solved with the help of global positioning systems (GLONASS, GPS). However, for the geohod and other mining machines working underground, the use of such systems is impossible because the satellite signal does not reach them. At present, positioning systems of miners and transports have become widespread in mines. The work of such systems is based on the detection of radio tags that are embedded in miners' cap lamps and mounted on the transport. Radio tags are detected with the help of readers which are placed in special wells drilled along the route of movement of miners and transport. Readers through the communication channels transmit information about the registered marks to the data collection server. It is obvious that the use of such systems for mining machines, including the geohod, is impossible, since readers and communication channels cannot be set in advance on the route of the machines. Thus, the development of a system of a geohod positioning in space is the subject for further research. The operation of such systems should be based on a combination of laser positioning technology, positioning by radio tags, orientation with the help of gyroscopes and computer modeling of the underground space.

Auxiliary systems of the geohod are systems ensuring the main process - the movement of the geohod in the geomedium. Such systems include: an energy-power device, a transport module and a lighting system. The task of control of these fundamentally different systems should also be solved by the geohod control system.

Difficult operating conditions of the geohod and a high level of dynamic loads predetermine special requirements to its reliability. One of the important factors of increasing the reliability in operation is technical diagnosis. The geohod control system should include a diagnostic subsystem that monitors the technical condition by measuring and indicating the parameters of the geohod elements, automation devices, electrical and hydraulic systems, lubrication systems, bearing assemblies, etc.

The main technical means for interaction of the geohod control system with the operator are the control panel and display. With the help of the control panel, the operator applies control actions to the subsystems, executive mechanisms and geohod devices working in manual, semi-automatic or automatic modes. It is necessary to solve the problem of a clear separation of control functions between the operator and the control device in semi-automatic mode. The display allows the operator to track in real time and retrospectively the status of processes and elements, emergency situations, the results of the system diagnostic. It is also possible to implement an additional sound and light operator alert, for example, to alert emergency situations. The task of the control system is to provide the operator with the necessary information.

\section{Results and discussion}

Taking into account the complexity and diversity of the formulated tasks concerning the geohod control, and taking into account the complex structure of the geohod, which consists 
of many interrelated systems, the general structure of the multilevel geohod control system, which is shown in figure 2., was developed.

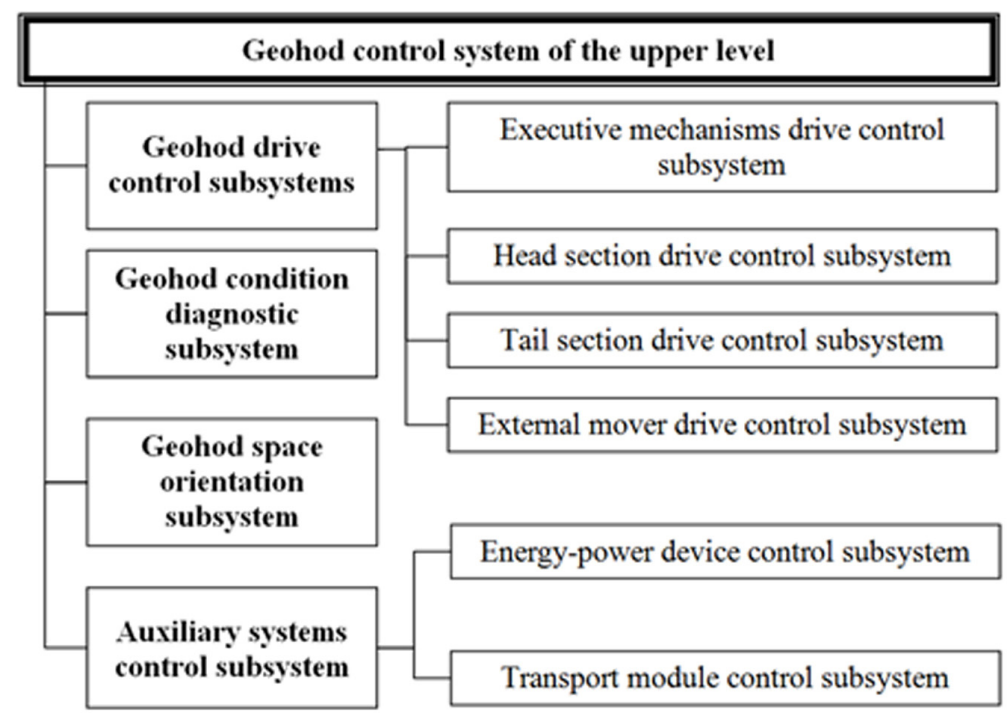

Fig. 2. General structure of the geohod control system.

The upper level control system is an information control system which solves the tasks of coordinating the work of subordinate subsystems, the exchange of information between these subsystems and the interaction of the geohod control system with the operator.

The middle level of the control system is represented by subsystems controlling drives, auxiliary systems, geohod diagnostics and its orientation in space.

Drive control subsystems solve the task of automatic control of executive mechanisms of geohod systems, ensuring the geohod movement in a changing geo-medium, taking into account the specified optimization criteria. The description of the tasks of other subsystems of the middle level is given above.

The lower level is represented by the subsystems controlling specific drives of the main (executive body, head and tail sections, external mover) and auxiliary (energy-power device, transport module) systems.

\section{Conclusion}

The above description of the tasks and the general structure of the control system make it possible to conclude that the tasks solved by the geohod control system are complex and diverse. However, at present there are no scientific approaches to develop mathematical models and algorithms of the geohod control systems. This inhibits the creation of the geohod with fundamentally new characteristics. Therefore, researches aimed at the solution of these tasks are actual.

To continue the research it is necessary:

- $\quad$ to formulate the requirements for the geohod control system;

- $\quad$ to analyze tasks solved by the geohod control system;

- develop the structure, mathematical models and algorithms for the operation of subsystems of the geohod control system;

- $\quad$ to develop software to simulate the interaction of subsystems of the geohod control system with each other, with the geomedium and the operator. 


\section{References}

1. R.V. Chernukhin, A.A. Dronov, M.Y. Blashchuk, IOP Conf. Ser.: Mater. Sci. Eng., 91, 012086 (2015)

2. A.B. Efremenkov, 6th International Forum on Strategic Technology, 2:1, 348 (2011).

3. V.Y. Begljakov, Timofeev V.Y., Dokhnenko M.V., Applied Mechanics and Materials, 682, 282 (2014).

4. G. Brierley, Y. Smith, World Tunnel. And subsurface Excav., 9 (1998)

5. J. Carmody, R. Sterling, Underground space design, (New York, 1993)

6. T. Wighman, ENR: News - Rec, 4 (1998)

7. S. Nishi, T. Seiki, Mem. Sch. Eng. Nagoya Univ., 1 (1997)

8. M.Y. Blashchuk, A.A. Kazantsev, R.V. Chernukhin, Applied Mechanics and Materials, 682, 418 (2014)

9. V. Aksenov, V. Sadovets, E. Rezanova, D. Pashkov E3S Web of Conferences, 15, 03015 (2017)

10. V.V. Aksenov, A.B. Efremenkov, V.Y. Beglyakov, Applied Mechanics and Materials, 379, 16 (2013).

11. M.Y. Blaschuk, A.A. Dronov, S.S. Ganovichev, IOP Conf. Ser. Mater. Sci. Eng., 127, 012033 (2016).

12. R.V. Chernukhin, M.Y. Blaschuk, V.Yu. Blumenstein, P.A. Chazov, IOP Conf. Ser. Mater. Sci. Eng., 142, 012003 (2016).

13. V.Y. Sadovets, V.Y. Beglyakov, A.B. Efremenkov, Applied Mechanics and Materials, 770, 384 (2015)

14. W.C. Zhong, J. Liu, M.Z. Xue, L.C. Jiao. IEEE Trans. On Systems, Man and Cybernetics, 34:2, 1128 (2004) 
